\title{
Efficacy and tolerability of lisdexamfetamine dimesylate in children with attention-deficit/ hyperactivity disorder: sex and age effects and effect size across the day
}

Sharon B Wigal ${ }^{1 *}$, Scott H Kollins², Ann C Childress ${ }^{3}$, Ben Adeyi ${ }^{4}$

\begin{abstract}
Background: Efficacy and safety profiles by sex and age (6-9 vs 10-12 years) and magnitude and duration of effect by effect size overall and across the day of lisdexamfetamine dimesylate (LDX) vs placebo were assessed.

Methods: This study enrolled children (6-12 years) with attention-deficit/hyperactivity disorder (ADHD) in an openlabel dose optimization with LDX (30-70 mg/d) followed by a randomized, double-blind, placebo-controlled, 2-way crossover phase. Post hoc analyses assessed interaction between sex or age and treatment and assessed effect sizes for Swanson, Kotkin, Agler, M-Flynn, and Pelham (SKAMP) and Permanent Product Measure of Performance (PERMP) scales and ADHD Rating Scale IV measures. No corrections for multiple testing were applied on time points and subgroup statistical comparisons.
\end{abstract}

Results: 129 participants enrolled; 117 randomized. Both sexes showed improvement on all assessments at postdose time points; females showed less impairment than males for SKAMP and PERMP scores in treatment and placebo groups at nearly all times. Both age groups improved on all assessments at postdose time points. Children 10-12 years had less impairment in SKAMP ratings than those 6-9 years. Treatment-by-sex interactions were observed at time points for SKAMP-D, SKAMP total, and PERMP scores; no consistent pattern across scales or time points was observed. LDX demonstrated significant improvement vs placebo, by effect size, on SKAMP-D from 1.5-13 hours postdose. The overall LS mean (SE) SKAMP-D effect size was -1.73 (0.18). In the dose-optimization phase, common $(22 \%)$ treatment-emergent adverse events (TEAEs) in males were upper abdominal pain, headache, affect lability, initial insomnia, and insomnia; in females were nausea and decreased weight. During the crossover phase for those taking LDX, higher incidence ( $22 \%$ greater) was observed in males for upper abdominal pain and insomnia and in females for nausea and headache. Overall incidence of TEAEs in age groups was similar.

Conclusion: Apparent differences in impairment level between sex and age groups were noted. However, these results support the efficacy of LDX from 1.5 hours to 13 hours postdose in boys and girls with medium to large effect sizes across the day with some variability in TEAE incidence by sex.

Trial Registration Number: ClinicalTrials.gov Identifier: NCT00500149.

\section{Background}

The efficacy and safety of stimulants for the pharmacologic management of attention-deficit/hyperactivity disorder (ADHD) is well documented [1,2]. Short-acting

\footnotetext{
* Correspondence: sbwigal@uci.edu

'University of California, Irvine, Child Development Center, Irvine, California, USA

Full list of author information is available at the end of the article
}

agents for the treatment of ADHD require multiple daily doses and have the potential for uneven symptom control $[3,4]$. After-school activities including sports or homework may last into later hours of the day, thus creating a need for long-acting stimulants for symptom control $[3,5]$. To address this and other limitations, novel delivery systems that result in longer durations of symptom control were developed [4-6].

\section{() Biomed Central}


Lisdexamfetamine dimesylate (LDX; Vyvanse ${ }^{\circ}$, Shire US Inc.) is a prodrug stimulant indicated for the treatment of ADHD in children (aged 6 to 12 years), adolescents (aged 13 to 17 years), and in adults. LDX is a therapeutically inactive molecule. After oral ingestion, LDX is converted to l-lysine and active d-amphetamine, which is responsible for the therapeutic effect [7]. In a 4-week, randomized, placebo-controlled, forced-dose titration trial in children with ADHD, LDX was administered in the morning with a median time of dose administration between 7:30 AM and 8:00 AM. LDX demonstrated efficacy versus placebo in improving ADHD symptoms by symptom ratings and global assessments from the first week of treatment through the end of the study [8]. In that study, LDX was well tolerated with a safety profile consistent with that of long-acting stimulant use. The most common adverse events (AEs) associated with LDX included decreased appetite, insomnia, abdominal pain, and irritability [8].

The onset and duration of efficacy of LDX in children was initially evaluated beginning 1 hour postdose and ending 12 hours postdose with significant efficacy shown from 2 to 12 hours [9]. A subsequent laboratory school study in children with ADHD evaluated onset and duration of efficacy from 1.5 to 13 hours postdose as measured by Swanson, Kotkin, Agler, M-Flynn, and Pelham (SKAMP) total and subscale scores. These results have been published elsewhere [10]. AEs in this study were consistent with those observed in other pediatric studies of LDX $[8,9]$ with the exception of a higher-than-typically-seen increase in pulse at 12.5 hours postdose for the participants receiving $70 \mathrm{mg} / \mathrm{d}$ LDX [10].

LDX has been shown to be generally effective for treating ADHD symptoms across the day. Despite this, little is known about the moderating effects of age and sex on treatment response to LDX or other stimulants, and existing studies report mixed results [11]. Results from the Multimodal Treatment Study of Children With ADHD (MTA), a large community-based trial of children 7.0 to 9.9 years of age, indicated an overall lack of a moderating effect of sex on treatment response $[12,13]$. However, an analysis of data from the Comparison of Methylphenidates in an Analog Classroom Setting (COMACS) study, a classroom analog study of children 6 to 12 years of age, found that females had a stronger response to methylphenidate from 1.5 to 3.0 hours postdose; from 4.5 to 6.0 hours, responses in males and females were equivalent, whereas from 7.5 to 12 hours, response among females declined more quickly than among males, leading to better response in males for those time points [14]. The COMACS study included 2 active treatment arms, osmotic-release oral methylphenidate (OROS-MPH) and methylphenidate extended-release (MPH-ER). Interestingly, although differences existed in the efficacy profiles of OROS-MPH and $\mathrm{MPH}-\mathrm{ER}$ in the overall group with MPH-ER demonstrating superiority early in the day and OROS$\mathrm{MPH}$ demonstrating superiority later in the day [6], the differences between males and females were independent of formulation [14].

Although some differential cognitive functioning has been found between girls and boys, most studies have documented little difference between sexes in cognitive and executive functions while clearly documenting significant impairment in these domains in both girls and boys with ADHD compared to children without ADHD [15]. Depression and anxiety may be more problematic in girls than in boys [16], whereas boys with ADHD are consistently reported to be more disruptive, more commonly involved in rule breaking, and more likely to have comorbid disruptive behavior disorders $[17,18]$.

Although the symptoms of ADHD may differ in patients of various age groups, little is known about differences in treatment effects and AEs experienced by children of different ages within the same study. In a study of preschool-aged children ( 3 to 5.5 years of age), treated with immediate-release methylphenidate in a 70week, multiple phase study utilizing both parent and teacher-rated assessments (the Preschool ADHD Treatment Study [PATS]), factor scores derived from the Conners, Loney, and Milich scale observed smaller effect sizes in parent and teacher ratings $(0.35$ and 0.43 , respectively) than those observed in results from the MTA study of school-aged children $(0.52$ and 0.75 , respectively) $[19,20]$. In addition, the preschoolers in the PATS showed a higher rate of methylphenidate discontinuation due to spontaneously reported AEs than did school-aged children in the MTA study (ie, $11 \%$ vs $<1 \%$ ) [21]. In fact, pharmacokinetic differences seem to corroborate other variables such as clearance rate contributing to age-related differences [22]. Although many factors may have contributed to these differences, they raise the question of whether certain age groups benefit more from treatment than do others.

Dosing of stimulants is not generally based on byweight guidelines, and younger, smaller children may receive relatively higher by-weight doses than do older, larger children. Younger, smaller children may theoretically be at higher risk for dose-dependent AEs. This possibility is supported by findings that these participants were prone to higher incidence of some AEs when receiving the highest dose level in a study of longacting methylphenidate [23]. Participants in the PATS experienced loss of appetite, trouble sleeping, stomachaches, social withdrawal, and lethargy, and these AEs occurred more frequently in participants receiving highdose methylphenidate than in those receiving low-dose 
methylphenidate or placebo [19]. Younger participants may be more likely to experience sleep difficulties and decreased appetite as AEs with stimulant treatment at high dose levels [23].

The post hoc analyses presented here assessed the efficacy of LDX in female and male participants and in younger (6 to 9 years) and older (10 to 12 years) participants to determine whether sex or age interactions were present. The safety profile of LDX was further characterized by examining AEs by sex and age. Also assessed was the duration of efficacy of LDX in a laboratory school setting based on effect size calculations for SKAMP and Permanent Product Measure of Performance (PERMP) measures. Effect size analyses are a useful method for providing clinically relevant information about the magnitude of effect relative to the effects of placebo, and where data are available, effect size assessments provide a systematic quantitative framework for assessing the relative effects of therapeutic agents across studies [24]. Effect size analyses may provide more practical information about the expected therapeutic effect (eg, efficacy and tolerability) that can be applied to making therapeutic choices.

\section{Study objectives}

The objective of this post hoc analysis was to examine the efficacy and safety profile of LDX by sex and age group in children with ADHD in a laboratory school setting. This analysis also aimed to assess the magnitude of effect overall and across the day of LDX vs placebo based on effect size analysis of SKAMP, PERMP, and ADHD Rating Scale IV (ADHD-RS-IV) scores.

\section{Methods}

This randomized, double-blind, multicenter, placebocontrolled, dose-optimization, crossover laboratory school study of LDX was conducted at 7 study sites in the United States. Full details of the methodological design and conduct of this study have been previously published [10]. All study activities were performed in accordance with the principles of the International Conference on Harmonisation Good Clinical Practice, 18th World Medical Assembly (Helsinki 1964), and amendments of the 29th (Tokyo 1975), the 35th (Venice 1983), the 41st (Hong Kong 1989), and the 48th (South Africa 1996) World Medical Assemblies. This was a study of children (6 to 12 years of age) diagnosed with moderate to severe ADHD (baseline ADHD-RS-IV score $\geq 28$ ) and included a screening and washout (for those participants taking other medications for ADHD at screening), an open-label, dose-optimization phase of $\operatorname{LDX}(30,50$, or $70 \mathrm{mg} / \mathrm{d}$ ) followed by a randomized, double-blind, placebo-controlled, 2-way crossover phase (Figure 1). Key exclusion criteria were the presence of a comorbid psychiatric condition with severe symptoms, conduct disorder, or other medical condition that could confound assessments, pose a risk to the participant, or prohibit study completion. Other inclusion and exclusion criteria were detailed previously [10].

\section{Efficacy measures}

Efficacy evaluations were performed on the intention-totreat population (ITT) population, defined as all participants who were randomized and had at least 1 SKAMP-Deportment (SKAMP-D) score available after randomization. Efficacy measures were collected in the

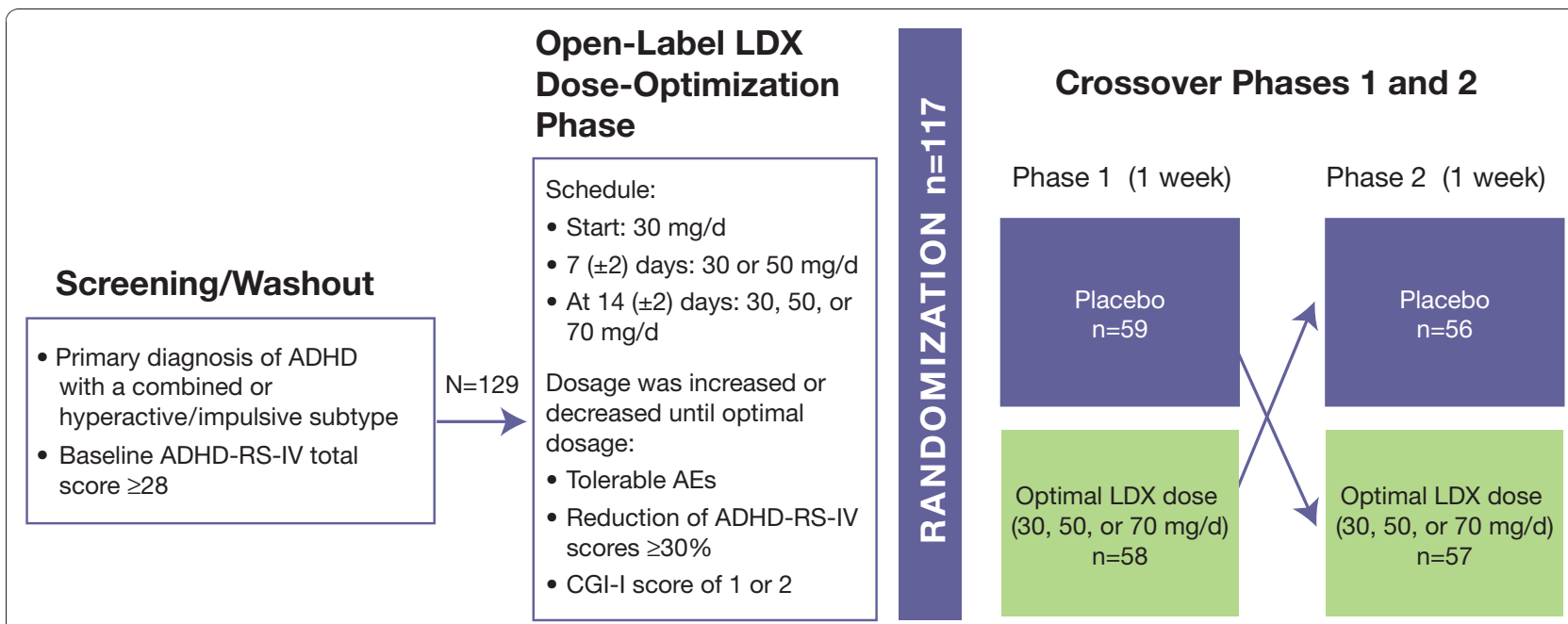

Figure 1 Study design. Twelve participants discontinued prior to randomization; 4 participants discontinued during the crossover period; 2 participants discontinued after the crossover phase; 9 participants discontinued because of AEs; no participants discontinued because of lack of efficacy. 
analog classroom setting at predose (-0.5 hours) and 1.5 , $2.5,5.0,7.5,10.0,12.0$, and 13.0 hours postdose during crossover periods 1 and 2 . The primary efficacy outcome was the onset of efficacy for LDX vs placebo as assessed by the primary outcome measure, SKAMP-D scores [10]. Key secondary assessments included the SKAMPAttention (SKAMP-A) and SKAMP quality of work subscales, SKAMP total scores, PERMP number attempted (PERMP-A) and PERMP number correct (PERMP-C), and the ADHD-RS-IV. SKAMP and PERMP were assessed predose and 1.5, 2.5, 5.0, 7.5, 10.0, 12.0, and 13.0 hours postdose during crossover periods 1 and 2 . ADHD-RS-IV was administered at baseline and during each weekly visit.

The SKAMP scale is a validated rating scale that assesses behavioral symptoms of ADHD in a classroom setting using a 7-point impairment scale $(0=$ none, $6=$ maximal impairment) $[25,26]$. The SKAMP total score comprises 13 items [26]. The SKAMP-D subscale evaluates deportment, including interacting with other children, interacting with adults, remaining quiet according to classroom rules, and staying seated according to classroom rules. The SKAMP-A subscale is a measure of attention and evaluates getting started on assignments, sticking with tasks, attending to an activity, and making activity transitions. The SKAMP quality of work subscale includes 3 items: completing assigned work, performing work accurately, and being careful and neat while writing or drawing.

The PERMP, a 5-page test consisting of 80 math problems per page (total of 400 problems) [26], evaluated effortful performance in the classroom as a measure of efficacy. Participants were instructed to work at their seats and to complete as many problems as possible in 10 minutes. The appropriate level of difficulty for each student was determined previously based on results of a math pretest administered at screening. Performance was evaluated using PERMP-A and PERMP-C scores.

The ADHD-RS-IV [27] is a clinician-rated scale that reflects current symptoms of ADHD based on Diagnostic and Statistical Manual of Mental Disorders, Fourth Edition, Text Revision (DSM-IV-TR) criteria; it is a global assessment that measures the severity of symptoms from visit to visit, but was not used to assess symptoms of ADHD over the course of the day. The ADHD-RS-IV consists of 18 items that are grouped into 2 subscales (hyperactivity/impulsivity and inattention). Each item is scored on a scale of 0 (no symptoms) to 3 (severe symptoms), yielding a total score of 0 to 54 .

\section{Safety assessments}

The safety population included all participants who were enrolled in the dose-optimization phase and received at least 1 dose of LDX. Treatment-emergent AEs (TEAEs), referring to events with onset after the first date of treatment, and no later than 3 days following termination of treatment, were recorded separately for the doseoptimization and the double-blind crossover phases of the study. TEAEs that continued uninterrupted from the dose-optimization to the crossover phase without a change in severity were counted only in the doseoptimization phase category. TEAEs with a change in severity across phases or that resolved and then restarted in the crossover phase were counted both in the doseoptimization and crossover arms. TEAEs for which a missing or incomplete start date made it impossible to determine in which phase of the study they started were counted as starting in the dose-optimization phase. TEAEs were reported as number and percentage of participants according to system-organ class, preferred term, treatment group, and by last dose received at $\mathrm{AE}$ onset. AEs were collected at all visits by soliciting participant report with nonleading questions, and were coded using the Medical Dictionary for Regulatory Activities (MedDRA).

\section{Statistical analyses}

Treatment interaction by age and sex were analyzed, post hoc, among the ITT population using a linear mixed model with sequence, period, sex (or age), treatment, and treatment by sex (or age) defined as fixed effects and subject-within-sequence as the random effect. No corrections for multiple testing were applied on time points and subgroup statistical comparisons.

Post hoc analyses evaluated SKAMP and PERMP effect size calculations for different dose groups, SKAMP and PERMP scores for males and females, and demographic data and AEs by age and sex. Least squares (LS) effect size and standard errors (SEs) were calculated according to the method of Curtin, Altman, and Elbourne [28] (standardized weighted mean difference [SWMD] methodology) at each postdose time point for SKAMP and PERMP assessments and for mean SKAMP-D score in the ITT population. The SWMD considers the treatment effect in relation to within-group standard deviation (SD) to combine continuous results of trials to evaluate the effect of treatment [28]. Effect size is a derived statistical assessment designed to allow comparisons of efficacy across clinical trials [29]. In general, effect size is calculated as the difference between drug effects and placebo effects divided by their pooled SD [29]. There are multiple methods for assessing pooled variance [28] depending on differences in the study design of included studies. Based on analysis by Cohen, effect sizes of $0.2,0.5$, and 0.8 , respectively, correspond to a small, medium, and large magnitude of effect [30]. Negative SKAMP effect sizes 
and positive PERMP effect sizes indicate improvement with LDX.

\section{Results}

During the dose-optimization phase, 58 participants were optimized to $30 \mathrm{mg} / \mathrm{d}$ LDX, 50 participants to $50 \mathrm{mg} / \mathrm{d}$ LDX, and 21 participants to $70 \mathrm{mg} / \mathrm{d}$ LDX; across all groups, participant demographics and characteristics were well balanced (Table 1). Overall demographic data have been published previously [10].

In the 2-way crossover phase of the study, 129 participants were enrolled and 117 were randomized. Eighteen participants discontinued the study with 9 discontinuing because of AEs. No participant discontinued because of lack of efficacy of LDX (Figure 1).

\section{Efficacy analyses}

Sex analysis

At the predose time point, there were significant effects of sex for SKAMP-D and SKAMP-A subscale scores and SKAMP total scores and significant treatment condition effects for SKAMP-A and SKAMP quality of work subscale scores and SKAMP total scores (Table 2). There were significant treatment condition effects for
PERMP-A and PERMP-C, and no significant effects of treatment-by-sex interactions were observed at the predose time point (Table 2).

Results of efficacy analyses for postdose time points by sex are shown in Figures 2 and 3 and mixed model statistical analysis in Table 2. There were significant effects of sex $(P<.05)$ at all time points for SKAMP-D scores. For SKAMP-D scores, the only significant treatment-bysex interaction was seen at the 7.5-hour time point. Results of the sex analysis for SKAMP total mirrored those of SKAMP-D with significant effects at all time points and a significant treatment-by-sex interaction at 7.5 and 10 hours postdose. For SKAMP-A, significant effects of sex were seen at only 1 time point (10 hours), and no significant treatment-by-sex interactions were observed at any postdose time point. For PERMP-A and PERMP-C, no significant effects of sex were seen, although significant treatment-by-sex interactions were seen at 1 time point (10 hours postdose). With LDX treatment, LS mean SKAMP scores for females were lower than those for males for all measures at all time points. Similarly, LS mean SKAMP scores for females were lower than those for males for all measures at all time points when receiving placebo.

Table 1 Participant Demographics (Safety Population)

\begin{tabular}{|c|c|c|c|c|c|c|c|}
\hline \multirow{2}{*}{ Category } & & \multirow[b]{2}{*}{ Age Group } & \multirow[b]{2}{*}{ Statistic } & \multicolumn{4}{|c|}{ LDX Dose } \\
\hline & & & & $30 \mathrm{mg} / \mathrm{d}$ & $50 \mathrm{mg} / \mathrm{d}$ & $70 \mathrm{mg} / \mathrm{d}$ & All doses \\
\hline \multirow[t]{4}{*}{ Age (y) } & & $6-9$ & $n$ & 27 & 15 & 6 & 48 \\
\hline & & & Mean (SD) & $8.5(0.75)$ & $8.5(0.74)$ & $8.0(0.89)$ & $8.5(0.77)$ \\
\hline & & $10-12$ & $n$ & 31 & 35 & 15 & 81 \\
\hline & & & Mean (SD) & $10.9(0.96)$ & $10.9(0.73)$ & $11.4(1.12)$ & $11.0(0.91)$ \\
\hline \multirow[t]{4}{*}{$\operatorname{Sex}^{*}$} & Male & $6-9$ & n (\%) & $18(66.7)$ & $13(86.7)$ & $6(100)$ & 37 (77.1) \\
\hline & Female & & n (\%) & $9(33.3)$ & $2(13.3)$ & 0 & $11(22.9)$ \\
\hline & Male & $10-12$ & n (\%) & 26 (83.9) & 24 (68.6) & 11 (73.3) & $61(75.3)$ \\
\hline & Female & & n (\%) & $5(16.1)$ & $11(31.4)$ & $4(26.7)$ & $20(24.7)$ \\
\hline \multirow[t]{4}{*}{ Weight (lb) } & & $6-9$ & $n$ & 27 & 15 & 6 & 48 \\
\hline & & & Mean (SD) & $62.2(8.78)$ & $65.0(13.75)$ & $53.1(2.50)$ & $61.9(10.62)$ \\
\hline & & $10-12$ & $n$ & 31 & 35 & 15 & 81 \\
\hline & & & Mean (SD) & $78.9(16.36)$ & 79.0 (18.64) & 80.7 (17.29) & 79.3 (17.34) \\
\hline \multirow[t]{4}{*}{ Height (in) } & & $6-9$ & $n$ & 27 & 15 & 6 & 48 \\
\hline & & & Mean (SD) & $51.2(2.30)$ & $52.1(2.29)$ & $49.3(2.04)$ & $51.3(2.38)$ \\
\hline & & $10-12$ & $n$ & 31 & 35 & 15 & 81 \\
\hline & & & Mean (SD) & $56.2(2.84)$ & $56.2(3.08)$ & $57.3(2.79)$ & $56.4(2.93)$ \\
\hline \multirow[t]{4}{*}{ Body mass index $\left(\mathrm{kg} / \mathrm{m}^{2}\right)$} & & $6-9$ & $n$ & 27 & 15 & 6 & 48 \\
\hline & & & Mean (SD) & $16.6(1.44)$ & 16.7 (2.38) & $15.4(1.28)$ & $16.5(1.78)$ \\
\hline & & 10-12 & $n$ & 31 & 35 & 15 & 81 \\
\hline & & & Mean (SD) & $17.4(2.45)$ & $17.4(2.67)$ & $17.2(2.55)$ & $17.4(2.53)$ \\
\hline
\end{tabular}

SD: standard deviation.

*Percentages are based on number of participants in the age group of the dose classification. 
Table 2 Mixed Model Analysis by Treatment, Sex, and Treatment by Sex for Predose and Postdose Time Points*

\begin{tabular}{|c|c|c|c|c|c|c|c|c|c|c|c|c|c|}
\hline \multirow{2}{*}{$\begin{array}{l}\text { Time Point } \\
\text { (hr) }\end{array}$} & \multirow{2}{*}{$\begin{array}{c}\text { Mixed Model Statistical } \\
\text { Analysis }\end{array}$} & \multicolumn{2}{|c|}{ SKAMP-D } & \multicolumn{2}{|c|}{ SKAMP-A } & \multicolumn{2}{|c|}{ SKAMP-Total } & \multicolumn{2}{|c|}{ SKAMP-QoL } & \multicolumn{2}{|c|}{ PERMP-A } & \multicolumn{2}{|c|}{ PERMP-C } \\
\hline & & $\begin{array}{c}F \\
\text { Value }\end{array}$ & $\begin{array}{c}P \\
\text { Value }\end{array}$ & $\begin{array}{c}F \\
\text { Value }\end{array}$ & $\begin{array}{c}P \\
\text { Value }\end{array}$ & $\begin{array}{c}F \\
\text { Value }\end{array}$ & $\begin{array}{c}P \\
\text { Value }\end{array}$ & $\begin{array}{c}F \\
\text { Value }\end{array}$ & $\begin{array}{c}P \\
\text { Value }\end{array}$ & $\begin{array}{c}F \\
\text { Value }\end{array}$ & $\begin{array}{c}P \\
\text { Value }\end{array}$ & $\begin{array}{c}\mathrm{F} \\
\text { Value }\end{array}$ & $\begin{array}{c}P \\
\text { Value }\end{array}$ \\
\hline \multirow[t]{3}{*}{-0.5} & Treatment & 0.99 & .3230 & 7.21 & .0084 & 28.52 & $<.0001$ & 93.12 & $<.0001$ & 22.19 & $<.0001$ & 23.58 & $<.0001$ \\
\hline & Sex & 9.36 & .0028 & 8.71 & .0039 & 13.38 & .0004 & 0.75 & .3891 & 0.31 & .5809 & 0.25 & .6164 \\
\hline & Treatment by sex & 1.95 & .1657 & 0.19 & .6620 & 2.49 & .1173 & 0.52 & .4714 & 0.59 & .4447 & 0.76 & .3861 \\
\hline \multirow[t]{3}{*}{1.5} & Treatment & 19.58 & $<.0001$ & 12.45 & .0006 & 28.01 & $<.0001$ & 1.06 & .3049 & 13.67 & .0003 & 19.73 & $<.0001$ \\
\hline & Sex & 6.59 & .0116 & 3.50 & .0639 & 7.84 & .0060 & 3.72 & .0562 & 0.37 & .5421 & 0.48 & .4899 \\
\hline & Treatment by sex & 0.00 & .9779 & 0.10 & .7528 & 0.20 & .6578 & 0.88 & .3493 & 0.05 & .8245 & 0.39 & .5315 \\
\hline \multirow[t]{3}{*}{2.5} & Treatment & 70.77 & $<.0001$ & 53.15 & $<.0001$ & 131.44 & $<.0001$ & 43.18 & $<.0001$ & 60.11 & $<.0001$ & 65.68 & $<.0001$ \\
\hline & Sex & 7.89 & .0059 & 2.35 & .1278 & 8.72 & .0038 & 2.88 & .0928 & 0.39 & .5326 & 0.39 & .5350 \\
\hline & Treatment by sex & 2.71 & .1023 & 0.81 & .3708 & 2.97 & .0875 & 0.15 & .6995 & 1.10 & .2975 & 1.62 & .2063 \\
\hline \multirow[t]{3}{*}{5.0} & Treatment & 74.56 & $<.0001$ & 50.75 & $<.0001$ & 136.60 & $<.0001$ & 68.23 & $<.0001$ & 73.30 & $<.0001$ & 76.40 & $<.0001$ \\
\hline & Sex & 7.13 & .0087 & 2.58 & .1111 & 7.00 & .0093 & 0.20 & .6518 & 0.23 & .6357 & 0.18 & .6708 \\
\hline & Treatment by sex & 2.50 & .1166 & 1.33 & .2517 & 1.63 & .2049 & 0.28 & .5949 & 1.18 & .2801 & 1.40 & .2398 \\
\hline \multirow[t]{3}{*}{7.5} & Treatment & 67.32 & $<.0001$ & 56.67 & $<.0001$ & 137.48 & $<.0001$ & 53.75 & $<.0001$ & 89.27 & $<.0001$ & 92.70 & $<.0001$ \\
\hline & Sex & 9.38 & .0028 & 3.52 & .0633 & 11.68 & .0009 & 10.46 & .0016 & 1.65 & .2021 & 1.73 & .1916 \\
\hline & Treatment by sex & 4.31 & .0402 & 2.10 & .1506 & 4.16 & .0438 & 0.24 & .6248 & 0.92 & .3406 & 0.76 & .3855 \\
\hline \multirow[t]{3}{*}{10} & Treatment & 44.74 & $<.0001$ & 39.70 & $<.0001$ & 92.89 & $<.0001$ & 17.42 & $<.0001$ & 64.41 & $<.0001$ & 69.95 & $<.0001$ \\
\hline & Sex & 8.67 & .0039 & 5.07 & .0263 & 13.35 & .0004 & 3.22 & .0754 & 0.65 & .4233 & 0.69 & .4083 \\
\hline & Treatment by sex & 1.51 & .2222 & 3.67 & .0579 & 6.31 & .0135 & 2.86 & .0939 & 5.43 & .0217 & 4.98 & .0277 \\
\hline \multirow[t]{3}{*}{12} & Treatment & 21.05 & $<.0001$ & 30.04 & $<.0001$ & 69.72 & $<.0001$ & 26.38 & $<.0001$ & 47.55 & $<.0001$ & 52.36 & $<.0001$ \\
\hline & Sex & 14.36 & .0002 & 2.33 & .1299 & 12.84 & .0005 & 0.67 & .4165 & 0.34 & .5613 & 0.36 & .5484 \\
\hline & Treatment by sex & 0.93 & .3374 & 0.37 & .5455 & 0.55 & .4586 & 0.31 & .5767 & 1.32 & .2524 & 1.42 & .2357 \\
\hline \multirow[t]{3}{*}{13} & Treatment & 3.70 & .0568 & 19.09 & $<.0001$ & 21.25 & $<.0001$ & 8.13 & .0052 & 39.39 & $<.0001$ & 41.15 & $<.0001$ \\
\hline & Sex & 12.45 & .0006 & 2.84 & .0945 & 13.56 & .0004 & 1.53 & .2187 & 1.08 & .3012 & 1.37 & .2439 \\
\hline & Treatment by sex & 1.30 & .2571 & 2.69 & .1039 & 3.03 & .0844 & 0.00 & .9942 & 3.58 & .0609 & 2.74 & .1006 \\
\hline
\end{tabular}

LDX: lisdexamfetamine dimesylate; PERMP: Permanent Product Measure of Performance; PERMP-A: PERMP-Attempted; PERMP-C: PERMP-Correct; SKAMP: Swanson, Kotkin, Agler, M-Flynn, and Pelham; SKAMP-A: SKAMP-Attention; SKAMP-D: SKAMP-Deportment.

*Degrees of freedom $(d f)=110$ for all analyses except the 7.5-hour postdose time point where $\mathrm{df}=109$ for all analyses.

\section{Age analysis}

At the predose time point, there were significant effects of age for SKAMP-D subscale and SKAMP total scores and significant treatment condition effects for SKAMPA subscale, SKAMP quality of work subscale, SKAMP total scores, PERMP-A, and PERMP-C (Table 3).

Results of efficacy analyses for postdose time points by age are shown in Figures 4 and 5 and mixed model statistical analysis in Table 3. There were significant effects of age at all time points for SKAMP-D subscale scores, except at the 10-hour time point, with participants aged 10 to 12 years showing less impairment than did those aged 6 to 9 years overall. Significant treatment-by-age interactions were seen at the 2.5- and 5-hour time points.

Results of age analysis for SKAMP total scores were similar to those of SKAMP-D and showed significant effects at all postdose time points, with participants aged 10 to 12 years demonstrating significantly less impairment than did those aged 6 to 9 years overall and a significant treatment-by-age interaction at 5 hours. For SKAMP-A, significant effects of age were seen at all postdose time points, except at 2.5 hours, and the only significant treatment-by-age interaction was observed at the 7.5-hour time point. With LDX treatment, LS mean SKAMP scores for participants aged 10 to 12 years were lower than were those for participants aged 6 to 9 years for all measures at all postdose time points with the exception of the SKAMP quality of work at the 7.5-hour time point. Similarly, LS mean SKAMP scores for participants aged 10 to 12 years were lower than those for participants aged 6 to 9 years for all measures at all postdose time points when receiving placebo, with the exception of the SKAMP quality of work subscale at the 1.5-, 10-, and 13-hour postdose time points. No significant effects for age were noted in PERMP-A and 

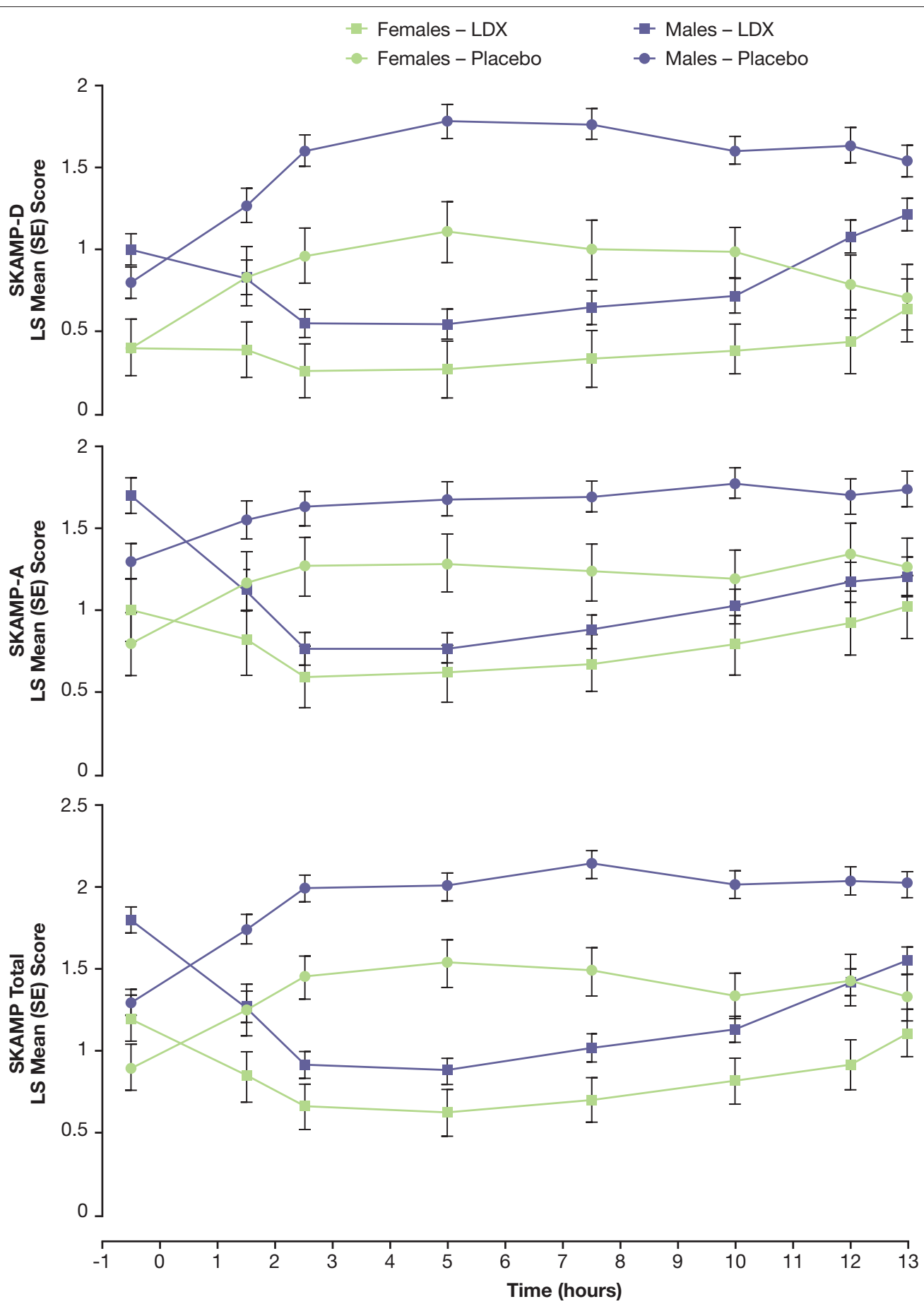

Figure 2 Postdose LS Mean (SE) SKAMP-D, SKAMP-A, and Total Scores by Time and Sex. Lower SKAMP scores indicate improvement.

PERMP-C analyses. Significant treatment-by-age interactions were noted at the 5-hour time point for both PERMP-A and PERMP-C measures.

\section{Effect size}

The predose LS mean (SE) effect size for the SKAMP-D subscale was $0.26(0.13)$. Based on effect sizes, LDX demonstrated significant improvement on the SKAMP$\mathrm{D}(P<.05)$ compared with placebo from 1.5 hours, the first postdose time point measured, to 13 hours postdose, the last time point measured. The LS mean (SE) treatment effect size over the classroom day on the SKAMP-D was -1.73 (0.18). The magnitude of effect 


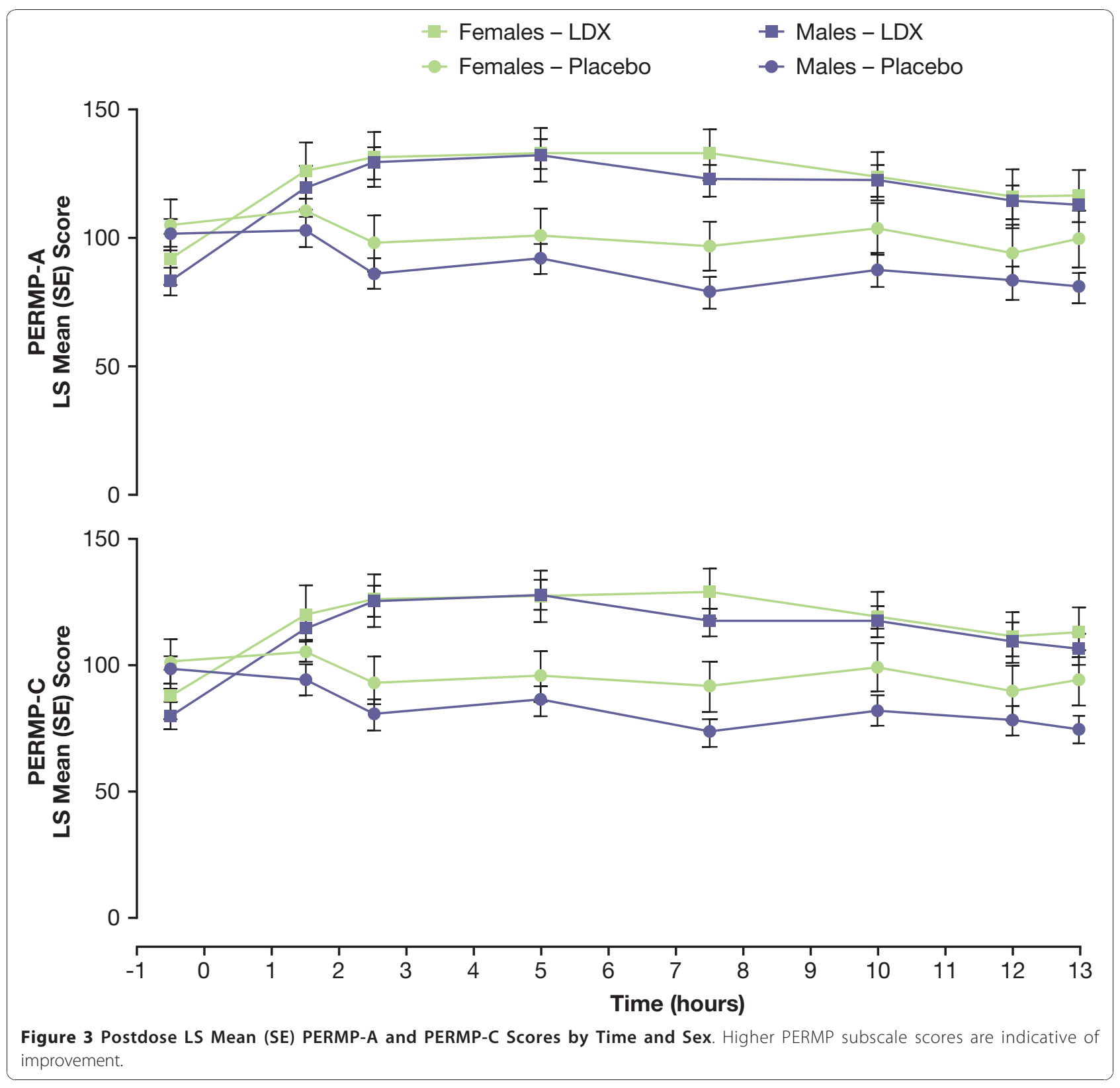

size of LDX treatment as measured by the SKAMP-D subscale was mostly medium to large except for the last time point (13 hours postdose) at which a small to medium effect was observed (Table 4).

The predose LS mean (SE) effect size for the SKAMPA and SKAMP quality of work subscales and SKAMP total scores were $0.45(0.14), 1.55(0.17)$, and $0.94(0.15)$, respectively. Based on effect sizes for SKAMP-A and SKAMP total scores, LDX demonstrated improvement compared with placebo from 1.5 hours to 13 hours postdose (Table 4); for SKAMP quality of work subscale, LDX demonstrated improvement compared with placebo from 2.5 hours to 13 hours postdose (Table 4).
The magnitude of effect size of LDX treatment as measured by SKAMP subscale scores (SKAMP-D, SKAMP-A, and SKAMP quality of work) and SKAMP total score effect sizes demonstrated a medium to large effect size of drug vs placebo at most postdose time points (Table 4).

The predose LS mean (SE) effect size for PERMP-A and PERMP-C scores were -0.79 (0.14) and -0.82 (0.14), respectively. The postdose effect size of LDX on PERMP-A and PERMP-C was large and maintained from 1.5 to 13 hours postdose (Table 4).

The mean raw postdose effect sizes for all optimized LDX dose groups $(30,50$, and $70 \mathrm{mg} / \mathrm{d})$ were mostly 
Table 3 Mixed Model Analysis by Treatment, Age, and Treatment by Age for Predose and Postdose Time Points*

\begin{tabular}{|c|c|c|c|c|c|c|c|c|c|c|c|c|c|}
\hline \multirow{2}{*}{$\begin{array}{l}\text { Time Point } \\
\text { (hr) }\end{array}$} & \multirow{2}{*}{$\begin{array}{c}\text { Mixed Model Statistical } \\
\text { Analysis }\end{array}$} & \multicolumn{2}{|c|}{ SKAMP-D } & \multicolumn{2}{|c|}{ SKAMP-A } & \multicolumn{2}{|c|}{ SKAMP-Total } & \multicolumn{2}{|c|}{ SKAMP-QoL } & \multicolumn{2}{|c|}{ PERMP-A } & \multicolumn{2}{|c|}{ PERMP-C } \\
\hline & & $\begin{array}{c}F \\
\text { Value }\end{array}$ & $\begin{array}{c}P \\
\text { Value }\end{array}$ & $\begin{array}{c}F \\
\text { Value }\end{array}$ & $\begin{array}{c}P \\
\text { Value }\end{array}$ & $\begin{array}{c}\mathrm{F} \\
\text { Value }\end{array}$ & $\begin{array}{c}P \\
\text { Value }\end{array}$ & $\begin{array}{c}F \\
\text { Value }\end{array}$ & $\begin{array}{c}P \\
\text { Value }\end{array}$ & $\begin{array}{c}\mathrm{F} \\
\text { Value }\end{array}$ & $\begin{array}{c}P \\
\text { Value }\end{array}$ & $\begin{array}{c}F \\
\text { Value }\end{array}$ & $\begin{array}{c}P \\
\text { Value }\end{array}$ \\
\hline \multirow[t]{3}{*}{-0.5} & Treatment & 3.69 & .0574 & 10.88 & .0013 & 48.67 & $<.0001$ & 133.44 & $<.0001$ & 34.98 & $<.0001$ & 37.63 & $<.0001$ \\
\hline & Age & 8.65 & .0040 & 3.55 & .0623 & 5.76 & .0181 & 0.95 & .3307 & 1.49 & .2251 & 1.60 & .2080 \\
\hline & Treatment by age & 0.10 & .7574 & 0.72 & .3971 & 0.31 & .5771 & 0.02 & .9027 & 0.20 & .6580 & 0.17 & .6793 \\
\hline \multirow[t]{3}{*}{1.5} & Treatment & 27.82 & $<.0001$ & 19.41 & $<.0001$ & 42.98 & $<.0001$ & 2.92 & .0903 & 18.95 & $<.0001$ & 30.00 & $<.0001$ \\
\hline & Age & 6.83 & .0102 & 6.63 & .0113 & 6.93 & .0097 & 0.03 & .8716 & 0.59 & .4430 & 0.54 & .4627 \\
\hline & Treatment by age & 2.38 & .1255 & 1.91 & .1693 & 2.32 & .1307 & 0.09 & .7625 & 0.49 & .4876 & 0.02 & .8854 \\
\hline \multirow[t]{3}{*}{2.5} & Treatment & 121.66 & $<.0001$ & 79.31 & $<.0001$ & 204.35 & $<.0001$ & 61.19 & $<.0001$ & 94.10 & $<.0001$ & 103.60 & $<.0001$ \\
\hline & Age & 7.25 & .0082 & 3.70 & .0571 & 7.99 & .0056 & 2.49 & .1172 & 0.14 & .7066 & 0.23 & .6303 \\
\hline & Treatment by age & 5.99 & .0159 & 0.01 & .9253 & 1.93 & .1672 & 0.02 & .8971 & 1.74 & .1902 & 1.27 & .2619 \\
\hline \multirow[t]{3}{*}{5.0} & Treatment & 126.22 & $<.0001$ & 79.64 & $<.0001$ & 213.18 & $<.0001$ & 89.58 & $<.0001$ & 117.59 & $<.0001$ & 122.97 & $<.0001$ \\
\hline & Age & 5.20 & .0245 & 6.59 & .0116 & 8.64 & .0040 & 2.49 & .1173 & 0.04 & .8480 & 0.07 & .7974 \\
\hline & Treatment by age & 5.57 & .0201 & 0.49 & .4865 & 4.54 & .0353 & 2.72 & .1018 & 4.41 & .0379 & 4.25 & .0415 \\
\hline \multirow[t]{3}{*}{7.5} & Treatment & 109.78 & $<.0001$ & 98.23 & $<.0001$ & 215.28 & $<.0001$ & 76.54 & $<.0001$ & 134.00 & $<.0001$ & 137.53 & $<.0001$ \\
\hline & Age & 5.27 & .0236 & 6.93 & .0097 & 7.27 & .0081 & 0.00 & .9885 & 0.21 & .6473 & 0.35 & .5544 \\
\hline & Treatment by age & 0.12 & .7342 & 6.55 & .0118 & 2.06 & .1543 & 0.06 & .8090 & 1.55 & .2159 & 1.38 & .2418 \\
\hline \multirow[t]{3}{*}{10} & Treatment & 72.09 & $<.0001$ & 68.52 & $<.0001$ & 150.65 & $<.0001$ & 32.26 & $<.0001$ & 108.63 & $<.0001$ & 115.45 & $<.0001$ \\
\hline & Age & 3.26 & .0736 & 11.15 & .0011 & 7.22 & .0083 & 0.23 & .6300 & 0.60 & .4393 & 0.64 & .4271 \\
\hline & Treatment by age & 0.83 & .3636 & 0.04 & .8433 & 0.05 & .8172 & 1.00 & .3186 & 0.20 & .6556 & 0.05 & .8202 \\
\hline \multirow[t]{3}{*}{12} & Treatment & 34.82 & $<.0001$ & 44.84 & $<.0001$ & 100.99 & $<.0001$ & 30.71 & $<.0001$ & 74.62 & $<.0001$ & 81.57 & $<.0001$ \\
\hline & Age & 5.34 & .0227 & 9.44 & .0027 & 12.67 & .0006 & 3.97 & .0487 & 0.13 & .7163 & 0.06 & .8065 \\
\hline & Treatment by age & 0.42 & .5170 & 0.06 & .8007 & 0.00 & .9549 & 1.06 & .3058 & 0.27 & .6020 & 0.13 & .7158 \\
\hline \multirow[t]{3}{*}{13} & Treatment & 8.29 & .0048 & 34.69 & $<.0001$ & 38.63 & $<.0001$ & 10.26 & .0018 & 67.42 & $<.0001$ & 68.29 & $<.0001$ \\
\hline & Age & 5.56 & .0201 & 7.40 & .0076 & 9.48 & .0026 & 0.34 & .5637 & 0.03 & .8639 & 0.09 & .7587 \\
\hline & Treatment by age & 0.01 & .9293 & 0.45 & .5018 & 0.45 & .5051 & 2.15 & .1458 & 0.02 & .8820 & 0.02 & .8913 \\
\hline
\end{tabular}

LDX: lisdexamfetamine dimesylate; PERMP: Permanent Product Measure of Performance; PERMP-A: PERMP-Attempted; PERMP-C: PERMP-Correct; SKAMP: Swanson, Kotkin, Agler, M-Flynn, and Pelham; SKAMP-A: SKAMP-Attention; SKAMP-D: SKAMP-Deportment.

*Degrees of freedom $(d f)=110$ for all analyses except the 7.5-hour postdose time point where $\mathrm{df}=109$ for all analyses.

large for SKAMP-D, SKAMP-A, and SKAMP quality of work subscales, and SKAMP total score (Table 5).

As previously reported, ADHD-RS-IV total score and ADHD-RS-IV inattention and hyperactivity/impulsivity subscale scores decreased from baseline for all doses of LDX during the dose-optimization phase and improved for all doses of LDX vs placebo (by difference in LS means: all $P<.0001)$ during the crossover phase [10]. Large treatment effect sizes were observed. The LS mean (SE) treatment effect size was -1.4 (0.16) for ADHD-RS-IV total score and -1.4 (0.16) for inattention and $-1.3(0.16)$ for hyperactivity/impulsivity subscale scores.

\section{Safety}

Overall safety data have been published previously [10]. There were no deaths or serious AEs reported during this study. Most TEAEs were mild to moderate in severity. During the dose-optimization phase, 110 participants (85.3\%) reported TEAEs; the most common TEAEs reported during this phase included decreased appetite $(47.3 \%)$, insomnia $(27.1 \%)$, headache (17.1\%), irritability (16.3\%), affect lability $(10.1 \%)$, and upper abdominal pain (15.5\%). During the crossover phase, the most common TEAEs reported for participants receiving LDX included decreased appetite (6.1\%), headache $(5.2 \%)$, and insomnia (4.3\%). Detailed vital signs and electrocardiographic (ECG) data were presented previously [10]. During the dose-optimization phase, there were small increases in systolic blood pressure (SBP), diastolic blood pressure (DBP), and pulse, but no dose-related changes were noted. During the crossover phase, small mean increases in SBP, DBP, and pulse were seen for participants while taking LDX and placebo. No clinically concerning trends in ECG parameters were identified.

The overall incidence of TEAEs in each sex was similar during the dose-optimization phase (Table 6). TEAEs 


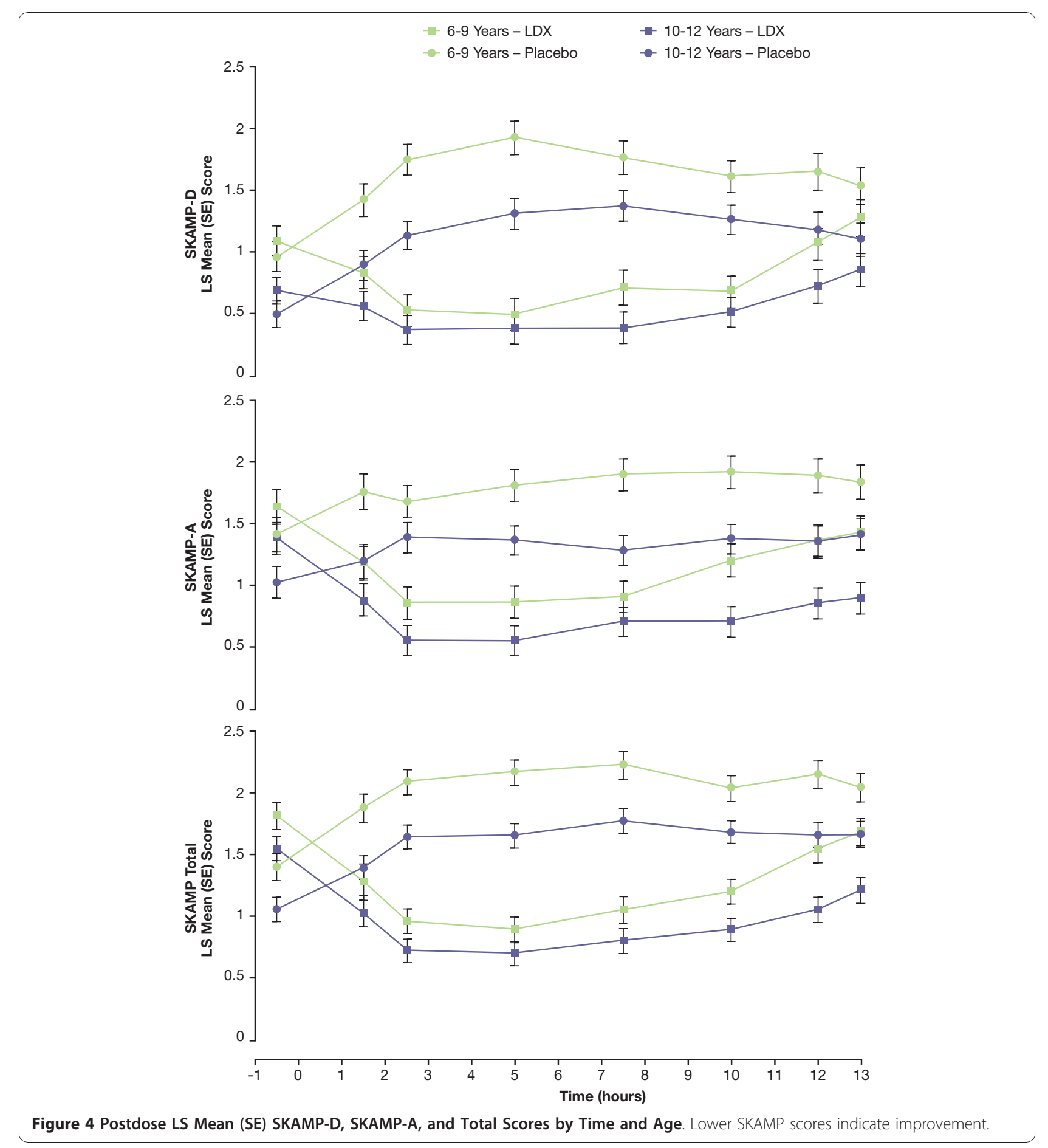

with $\geq 2 \%$ difference between sexes in the dose-optimization phase included upper abdominal pain (males, $16.3 \%$; females, $12.9 \%$ ), nausea (males, $7.1 \%$; females, $12.9 \%$ ), decreased weight (males, $2.0 \%$; females, $6.5 \%$ ), headache (males, $18.4 \%$; females, $12.9 \%$ ), affect lability (males, 11.2\%; females, 6.5\%), initial insomnia (males, $6.1 \%$; females, $0.0 \%$ ), and insomnia (males, $30.6 \%$; females, 16.1\%). During the crossover phase (Table 7), when receiving LDX, males had a numerically greater rate of TEAEs (males, 34.5\%; females, 28.6\%). Differences $(\geq 2 \%)$ in rates of TEAEs between sexes when receiving $\mathrm{LDX}$ in the crossover phase were upper abdominal pain (males, $2.3 \%$; females, $0.0 \%$ ), nausea (males, $1.1 \%$; females, $3.6 \%$ ), headache (males, $4.6 \%$; 


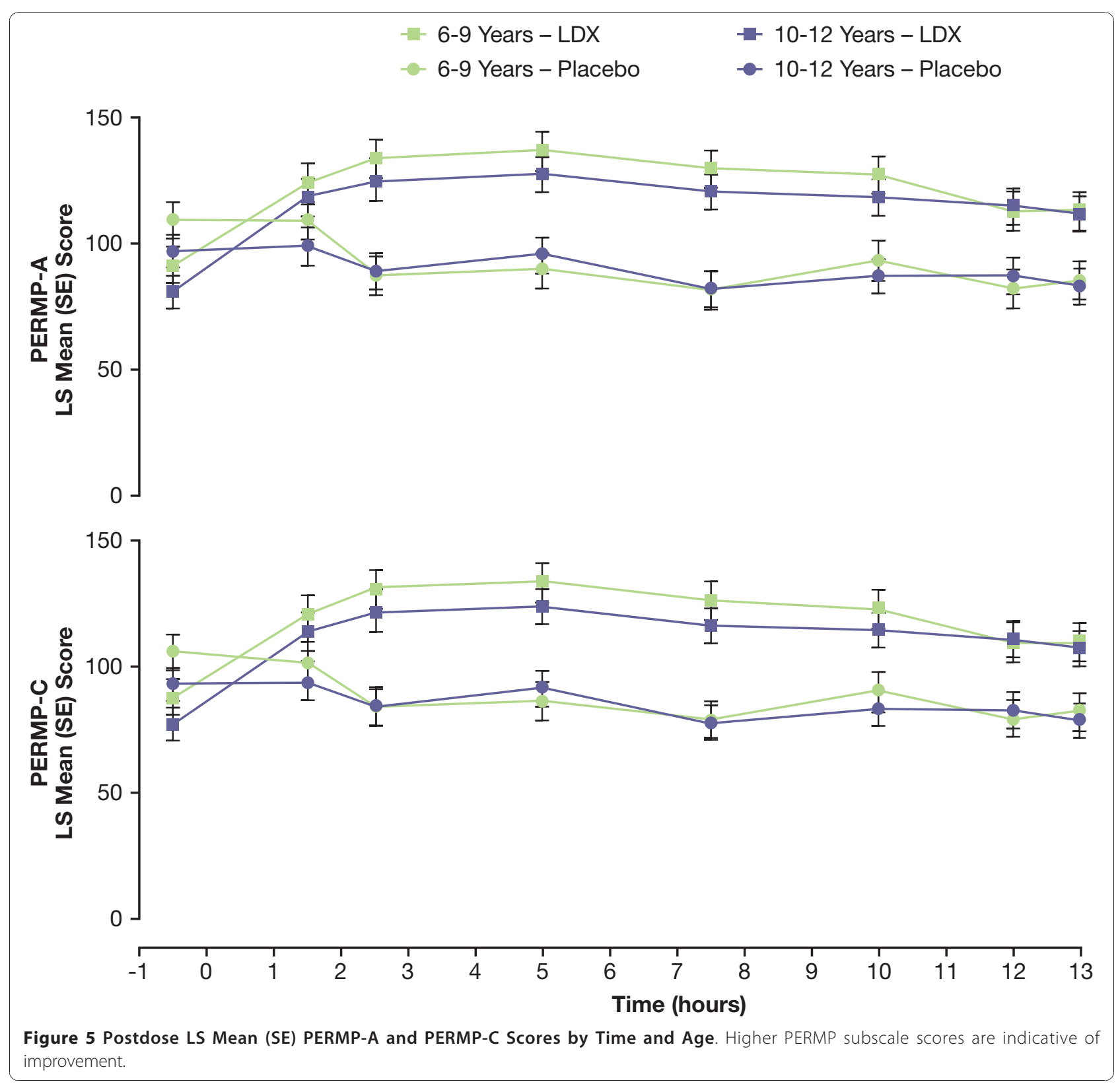

females, 7.1\%), and insomnia (males, 5.7\%; females, $0.0 \%)$.

Male participants receiving LDX had mean (SD) changes in weight from baseline of -1.5 (6.8), -2.0 (6.4), -2.6 (5.7), -2.1 (7.8), and -2.7 (6.2) lb at weeks 1, 2, 3, 4, and $5 / 6$, respectively. Female participants receiving LDX had mean (SD) changes in weight from baseline of -1.9 (2.5), -2.3 (1.9), -3.4 (2.0), -3.5 (2.5), and -2.1 (7.0) lb at weeks $1,2,3,4$, and $5 / 6$, respectively.

The overall incidence of TEAEs in each age group was similar during dose-optimization and crossover phases (Tables 8 and 9). In the dose-optimization phase, the incidence rates by dose group at the time of first occurrence of anorexia in the younger group were $10.4 \%, 0.0 \%$, and $0.0 \%$ and in the older group were $1.2 \%$, $0.0 \%$, and $6.3 \%$ for participants receiving 30,50 , and 70 $\mathrm{mg} / \mathrm{d}$ LDX, respectively. The incidence rates by dose group at the time of first occurrence of decreased appetite were $37.5 \%, 17.4 \%$, and $14.3 \%$ in the younger group and $42.0 \%, 7.8 \%$, and $0.0 \%$ in the older group, for participants receiving 30,50 , and $70 \mathrm{mg} / \mathrm{d} \mathrm{LDX}$, respectively. In the dose-optimization phase, the incidence rates by dose group at the time of first occurrence of insomnia were $31.3 \%, 13.0 \%$, and $0.0 \%$ in the younger group and $17.3 \%, 7.8 \%$, and $0.0 \%$ in the older group, for participants receiving 30,50 , and $70 \mathrm{mg} / \mathrm{d} \mathrm{LDX}$, respectively. 
Table 4 LS Mean (SE) Postdose SKAMP and PERMP Effect Sizes*

\begin{tabular}{lcccccc}
\hline Hour & $\begin{array}{c}\text { SKAMP-D LS Mean } \\
\text { (SE) Effect Size }\end{array}$ & $\begin{array}{c}\text { SKAMP-A LS Mean } \\
\text { (SE) Effect Size }\end{array}$ & $\begin{array}{c}\text { SKAMP Quality of Work LS } \\
\text { Mean (SE) Effect Size }\end{array}$ & $\begin{array}{c}\text { SKAMP Total LS } \\
\text { Mean (SE) Effect } \\
\text { Size }\end{array}$ & $\begin{array}{c}\text { PERMP-A LS Mean } \\
\text { (SE) Effect Size }\end{array}$ & $\begin{array}{c}\text { PERMP-C LS Mean } \\
\text { (SE) Effect Size }\end{array}$ \\
\hline 1.5 & $-0.68(0.14)$ & $-0.57(0.14)$ & $-0.23(0.13)$ & $-0.85(0.15)$ & $0.59(0.14)$ & $0.74(0.14)$ \\
\hline 2.5 & $-1.41(0.16)$ & $-1.20(0.16)$ & $-1.05(0.15)$ & $-1.89(0.18)$ & $1.28(0.16)$ & $1.34(0.16)$ \\
\hline 5.0 & $-1.44(0.16)$ & $-1.19(0.16)$ & $-1.24(0.16)$ & $-1.90(0.18)$ & $1.40(0.16)$ & $1.44(0.16)$ \\
\hline 7.5 & $-1.41(0.16)$ & $-1.27(0.16)$ & $-1.17(0.16)$ & $-1.94(0.19)$ & $1.53(0.17)$ & $1.56(0.17)$ \\
\hline 10.0 & $-1.12(0.15)$ & $-1.11(0.15)$ & $-0.77(0.14)$ & $-1.64(0.17)$ & $1.39(0.16)$ & $1.44(0.16)$ \\
\hline 12.0 & $-0.78(0.14)$ & $-0.89(0.15)$ & $-0.75(0.14)$ & $-1.35(0.16)$ & $1.15(0.15)$ & $1.21(0.16)$ \\
\hline 13.0 & $-0.38(0.14)$ & $-0.80(0.14)$ & $-0.44(0.14)$ & $-0.84(0.14)$ & $1.10(0.15)$ & $1.11(0.15)$ \\
\hline Mean ${ }^{\dagger}$ & $-1.73(0.18)$ & $-1.54(0.17)$ & $-1.73(0.18)$ & $-2.41(0.21)$ & $1.78(0.18)$ & $1.83(0.18)$ \\
\hline
\end{tabular}

LS: least squares; PERMP: Permanent Product Measure of Performance; PERMP-A: PERMP-Attempted; PERMP-C: PERMP-Correct; SE: standard error; SKAMP: Swanson, Kotkin, Agler, M-Flynn, and Pelham; SKAMP-A: SKAMP-Attention; SKAMP-D: SKAMP-Deportment.

*Negative SKAMP effect sizes indicate improvement with LDX. Positive PERMP effect sizes indicate improvement with LDX.

${ }^{\dagger}$ Mean SKAMP and PERMP scores across the day were calculated employing the model-based calculations using all measures for all participants at all time points across the day.

Participants aged 6 to 9 years receiving LDX had mean changes in weight from baseline of -2.5 (6.8), -2.3 (8.1), -3.4 (7.2), $-2.2(9.8)$, and $-2.9(8.8) \mathrm{lb}$ at weeks 1,2 , 3,4 , and $5 / 6$, respectively. At week $5 / 6$, the mean (SD) weight loss was -0.7 (6.5), -6.6 (11.8), and -1.4 (1.5) lb for younger participants receiving 30, 50, $70 \mathrm{mg} / \mathrm{d} \mathrm{LDX}$, respectively. Participants aged 10 to 12 years receiving LDX had mean (SD) changes in weight from baseline of $-0.8(5.1),-1.9(2.0),-2.3(2.1),-2.6(2.5)$, and $-2.3(3.0)$ $\mathrm{lb}$ at weeks $1,2,3,4$, and 5/6, respectively. At week 5/6, the mean (SD) weight loss of participants was -1.7 (3.2), -2.3 (2.4), and -3.1 (3.8) $\mathrm{lb}$ for older participants receiving $30,50,70 \mathrm{mg} / \mathrm{d} \mathrm{LDX}$, respectively.

\section{Discussion}

\section{Effects of Sex}

The effects of sex on treatment response have not been thoroughly studied; however, some sex differences in psychosocial, cognitive, and psychiatric functioning have been noted and may influence response to treatment. Psychosocial functioning in boys and girls is generally similar, but more externalizing behaviors have been identified in boys [16]. Existing data on sex as a moderating factor in treatment response are mixed [11]. In addition to the above clinical data, recent studies in animal models [31] and healthy human adults [32] suggest the potential for sex differences in the physiologic response to agents that interact with the dopaminergic system in the brain. The estrous cycle in female rats modulates amphetamine-stimulated dopamine release and other dopaminergic functions in the striatum [31]. Along similar lines, using brain imaging techniques, Munro et al demonstrated that males had greater amphetamine-stimulated dopamine release in the ventral striatum, anterior putamen, as well as the anterior and posterior caudate nuclei [32]. Additionally, subjective positive effects of amphetamine were greater in men than in women [32]. These preliminary findings are interesting; however, additional clinical and nonclinical studies will be necessary to determine whether these differences in brain physiology will result in demonstrable differences in clinical response.

In the current study, significant effects of sex were noted for SKAMP-D and SKAMP total scores. These effects were not noted for SKAMP-A, SKAMP quality of work, PERMP-A, or PERMP-C. Significant treatmentby-sex interactions were not seen at the majority of time points. Females in both placebo and treatment conditions had lower (ie, improved) scores on all SKAMP total and subscale scores than did males at all time points. While receiving placebo, females had higher (less impaired) scores on the PERMP-A and PERMP-C than did males receiving placebo. With LDX treatment, females and males improved and had similar scores on

Table 5 Postdose SKAMP Mean (SE) Effect Size* by Optimized Dose

\begin{tabular}{ccccc}
\hline LDX Dose Group & SKAMP-D & SKAMP-A & SKAMP Quality of Work & SKAMP Total \\
\hline $30 \mathrm{mg} / \mathrm{d}(\mathrm{n}=46)$ & $-0.85(0.23)$ & $-0.67(0.22)$ & $-0.94(0.23)$ & $-1.13(0.24)$ \\
\hline $50 \mathrm{mg} / \mathrm{d}(\mathrm{n}=47)$ & $-0.86(0.22)$ & $-0.80(0.22)$ & $-1.14(0.24)$ & $-1.18(0.24)$ \\
\hline $70 \mathrm{mg} / \mathrm{d}(\mathrm{n}=20)$ & $-1.14(0.36)$ & $-0.98(0.35)$ & $-0.83(0.34)$ & $-1.24(0.37)$ \\
\hline
\end{tabular}

LDX: lisdexamfetamine dimesylate; SE: standard error; SKAMP: Swanson, Kotkin, Agler, M-Flynn, and Pelham; SKAMP-A: SKAMP-Attention; SKAMP-D: SKAMPDeportment.

*Negative effect sizes indicate improvement with LDX. 
Table 6 TEAEs by sex in the dose-optimization phase while receiving LDX with an incidence of $\geq 10 \%$ in the dose-optimization and/or crossover phase

\begin{tabular}{|c|c|c|}
\hline \multirow[t]{4}{*}{ AE-Preferred Term } & \multicolumn{2}{|c|}{ Dose-Optimization Phase } \\
\hline & Males & Females \\
\hline & $(n=98)$ & $(n=31)$ \\
\hline & n (\%) & n (\%) \\
\hline Any AE & $83(84.7)$ & $27(87.1)$ \\
\hline Abdominal pain upper & $16(16.3)$ & $4(12.9)$ \\
\hline Affect lability & $11(11.2)$ & $2(6.5)$ \\
\hline Decreased appetite & $47(48.0)$ & $14(45.2)$ \\
\hline Headache & $18(18.4)$ & $4(12.9)$ \\
\hline Insomnia & $30(30.6)$ & $5(16.1)$ \\
\hline Irritability & $16(16.3)$ & $5(16.1)$ \\
\hline Nausea & $7(7.1)$ & $4(12.9)$ \\
\hline \multicolumn{3}{|c|}{$\begin{array}{l}\text { For tables 6-9: TEAEs were assigned to either the open-label dose- } \\
\text { optimization phase or the double-blind crossover phase of the study and } \\
\text { were summarized separately. TEAEs that continued uninterrupted from the } \\
\text { dose-optimization to the crossover phase without a change in severity were } \\
\text { counted only in the dose-optimization phase category. TEAEs with a change } \\
\text { in severity across phases or that resolved and then restarted in the crossover } \\
\text { phase were counted both in the dose-optimization and crossover arms. TEAEs } \\
\text { for which a missing or incomplete start date made it impossible to determine } \\
\text { in which phase of the study they started were counted as starting in the } \\
\text { dose-optimization phase. }\end{array}$} \\
\hline
\end{tabular}

PERMP-A and PERMP-C. The lack of consistent treatment-by-sex interactions indicated that both males and females responded well to LDX. Further, the results of this analysis support the efficacy of LDX from 1.5 hours to 13 hours postdose in both male and female participants. The duration of effect of LDX does not seem to differ between males and females. Although there appeared to be greater separation between the LDX and placebo conditions among males, females generally showed less impairment prior and subsequent to dosing than did males in the same treatment condition. The beneficial effects of LDX observed may have been

Table 7 TEAEs by sex in the crossover phase while receiving $L D X$ with an incidence of $\geq 10 \%$ in the dose-optimization and/or crossover phase

\begin{tabular}{lcc}
\hline AE-Preferred Term & \multicolumn{2}{c}{ Crossover Phase } \\
\hline & $\begin{array}{c}\text { Males } \\
(\mathrm{n}=87)\end{array}$ & $\begin{array}{c}\text { Females } \\
(\mathrm{n}=28)\end{array}$ \\
\hline $\mathrm{n}(\%)$ & $\mathrm{n}(\%)$ \\
\hline Any AE & $30(34.5)$ & $8(28.6)$ \\
\hline Abdominal pain upper & $2(2.3)$ & $0(0.0)$ \\
\hline Affect lability & $0(0.0)$ & $0(0.0)$ \\
\hline Decreased appetite & $5(5.7)$ & $2(7.1)$ \\
\hline Headache & $4(4.6)$ & $2(7.1)$ \\
\hline Insomnia & $5(5.7)$ & $0(0.0)$ \\
\hline Irritability & $1(1.1)$ & $0(0.0)$ \\
\hline Nausea & $1(1.1)$ & $1(3.6)$ \\
\hline
\end{tabular}

Table 8 TEAEs by age group in the dose-optimization phase while receiving LDX with an incidence of $\geq 10 \%$ in the dose-optimization and/or crossover phase

\begin{tabular}{lcc}
\hline AE-Preferred Term & \multicolumn{2}{c}{ Dose-Optimization Phase } \\
\hline $\begin{array}{c}6 \text { to } 9 \text { year olds } \\
(\mathrm{n}=48) \\
\mathrm{n}(\%)\end{array}$ & $\begin{array}{c}10 \text { to } 12 \text { year olds } \\
(\mathrm{n}=81)\end{array}$ & $\mathrm{n}(\%)$ \\
\hline Any AE & $43(89.6)$ & $67(82.7)$ \\
\hline Abdominal pain upper & $8(16.7)$ & $12(14.8)$ \\
\hline Affect lability & $7(14.6)$ & $6(7.4)$ \\
\hline Anorexia & $5(10.4)$ & $2(2.5)$ \\
\hline Decreased appetite & $23(47.9)$ & $38(46.9)$ \\
\hline Headache & $9(18.8)$ & $13(16.0)$ \\
\hline Insomnia & $17(35.4)$ & $18(22.2)$ \\
\hline Irritability & $7(14.6)$ & $14(17.3)$ \\
\hline
\end{tabular}

restricted by the limited impairment seen in females overall (ie, a floor effect). This finding is similar to that in the COMACS study [14], an analog classroom study of male and female children that used typical laboratory school measures to assess once-daily methylphenidate vs placebo but that likewise was not powered statistically to specifically measure student sex-based differences in stimulant treatment.

However, the results of the post hoc treatment-by-sex analysis presented here differs from the COMACS study, a trial with a similar double-blind, analog classroom design and a similar participant age profile, in which efficacy at early vs later time points differed between males and females [14]. Additionally, statistical analysis of SKAMP scores in the placebo cohort showed differences at predose and most postdose time points between males and females [14]. Interestingly, differences between sexes were not apparent when assessing PERMP scores in the COMACS study [14]. While no

Table 9 TEAEs by age group in the crossover phase while receiving LDX with an incidence of $\geq 10 \%$ in the dose-optimization and/or crossover phase

\begin{tabular}{lcc}
\hline AE-Preferred Term & \multicolumn{2}{c}{ Crossover Phase } \\
\hline $\begin{array}{c}6 \text { to } 9 \text { year olds } \\
(\mathrm{n}=42) \\
\mathrm{n}(\%)\end{array}$ & $\begin{array}{c}10 \text { to } 12 \text { year olds } \\
(\mathrm{n}=73)\end{array}$ & $\mathrm{n}(\%)$ \\
\hline Any AE & $16(38.1)$ & $22(30.1)$ \\
\hline Abdominal pain upper & $1(2.4)$ & $1(1.4)$ \\
\hline Affect lability & $0(0.0)$ & $0(0.0)$ \\
\hline Anorexia & $0(0.0)$ & $0(0.0)$ \\
\hline Decreased appetite & $2(4.8)$ & $5(6.8)$ \\
\hline Headache & $2(4.8)$ & $4(5.5)$ \\
\hline Insomnia & $3(7.1)$ & $2(2.7)$ \\
\hline Irritability & $1(2.4)$ & $0(0.0)$
\end{tabular}


statistical analysis was performed here to assess differences between sexes for the placebo cohort alone, the current data similarly appear to show differential characteristics between sexes in the placebo cohort for SKAMP but not PERMP measures. While these sex differences are intriguing, any consideration of cause would be speculative in nature. These findings indicate a need for continued close examination of differential presentation of ADHD symptoms and symptom clusters in females and males.

Although the distribution of participants by sex and racial group was similar, there were some differences between the current study and the COMACS study in participant demographic characteristics. Mean age was slightly higher in the current study than in the COMACS study (10.1 vs 9.58 years), and all participants in the current study had combined-type ADHD, whereas approximately $82 \%$ in the COMACS study had combined-type ADHD [14]. Although the application of different entry criteria (ADHD-RS-IV scores $\geq 28$ vs no severity cutoff) and baseline ADHD assessment measures (ADHD-RS-IV vs Swanson, Nolan and Pelham scale [SNAP]) in the current study and the COMACS study, respectively, make it difficult to specifically assess similarity in baseline ADHD symptom severity [14], overall SNAP baseline inattention and overactivity/ impulsivity scores between 1.09 and 1.28 suggest that ADHD severity in the COMACS study population may have been somewhat less than the severity in the current study (mean ADHD-RS-IV baseline score was 42.4).

\section{Effects of age}

Significant effects for age were noted in SKAMP-D, SKAMP-A, and SKAMP total scores, but not for PERMP-A or PERMP-C measures. Significant treatment-by-age interactions were not seen at the majority of time points for both SKAMP and PERMP measures. Both age groups responded to treatment. Older children in both the placebo and the treatment condition groups had lower SKAMP total and subscale scores than did younger children at predose and in a majority of the measured postdose time points. These findings of lower scores suggesting less impairment in older than younger children are in general agreement with previous findings that highlight age-dependent decline of symptoms and age-dependent changes in the functional expression of symptoms of ADHD [33-35]. The duration of effect of LDX does not seem to differ between age groups.

\section{Effect size}

From 1.5 to 13 hours postdose, the effect size of LDX for the primary efficacy variable, SKAMP-D, was medium to large at most postdose time points, as was the mean effect size over the course of the day. It should be noted that overall mean effect sizes across the day for SKAMP-D and the other SKAMP and PERMP measures were calculated in the model-based system and included all appropriate assessment values for all time points, so variance of these calculated means is commensurately small. The mean overall raw effect sizes for all optimized doses of LDX (median optimized LDX dose was $50 \mathrm{mg} / \mathrm{d}$ ) were medium to large for SKAMP-D, SKAMP-A, SKAMP quality of work, and SKAMP total scores. These results are not indicative of dose response because participants were not randomized to a dose group but rather were clinically titrated to LDX doses studied in the double-blind portion of the study. Thus, in the analog classroom setting, LDX was effective in reducing ADHD symptoms of deportment and attention from 1.5 to 13 hours postdose based on optimized dosing with mostly medium to large effect sizes for a broad array of assessments, including clinician observations, classroom behavior, and test of math performance.

Large treatment effect sizes were also observed for DSM-IV-TR-defined ADHD symptoms as assessed by ADHD-RS-IV total and subscores. These findings are consistent with a previous report that demonstrated similarly large ADHD-RS-IV effect sizes (1.39, 1.42, and 1.73 for 30, 50, and $70 \mathrm{mg} / \mathrm{d}$ LDX, respectively) in children with ADHD receiving LDX in a clinical trial with a longitudinal design [36].

Meta-analyses of multiple studies have concluded that effect sizes for the treatment of ADHD average 0.91 to 0.95 for stimulant agents [24,37]. A randomized, doubleblind, placebo-controlled study of the MPH-ER formulation $(20,40$, or $60 \mathrm{mg} / \mathrm{d})$ and OROS-MPH formulation $(18,36$, or $54 \mathrm{mg} / \mathrm{d})$ in the treatment of ADHD in children (6-12 years of age) examined effect sizes at various time points. Dosing was based on preexisting methylphenidate dosing. Effect sizes for MPH-ER as measured by SKAMP-D ranged from 0.06 (at 12 hours postdose) to 0.89 (at 3 hours postdose). Effect sizes for OROS$\mathrm{MPH}$ ranged from 0.25 (at 12 hours postdose) to 0.66 (at 6 hours postdose) [6]. A placebo-controlled, doubleblind, multiple-crossover study of methylphenidate (approximately $0.3 \mathrm{mg} / \mathrm{kg} / \mathrm{d}$ ) that studied the same participants as children and as adolescents in a summer treatment program reported an overall effect size, the product of 12 different measures (including IOWA Conners Rating Scale-counselor and IOWA Conners Rating Scale-teacher ratings, assessment of classwork completed and correct, and behavioral assessments of rule violations and other negative and positive behaviors), of 0.82 in children and 0.59 in adolescents [38].

Small to medium effect sizes favoring placebo were observed at predose time points for SKAMP-A and SKAMP-D, and large effect sizes at predose favoring placebo were observed for SKAMP quality of work, 
SKAMP total, PERMP-A, and PERMP-C scales. Although many factors may have contributed to this observation, residual drug from the previous treatment day may have played a role in this finding as has been seen in another study with an amphetamine-based longacting stimulant [39]. Pharmacokinetic data showing residual plasma levels were measurable prior to dosing with long-acting stimulants $[40,41]$. The impact of these pretreatment differences on the ultimate relationship between postdose LDX and placebo responses is unclear.

Overall incidence of TEAEs in this study has been described in detail [10] and is generally similar to that reported in other pediatric studies of LDX [8] and other long-acting stimulants $[9,42,43]$ where active treatment was begun after a washout period for prior treatments. A consistent pattern of greater incidence of TEAEs in either age group did not emerge. The incidence of anorexia was higher in the younger group than in the older group. Although the incidence of decreased appetite was roughly equivalent, the greater incidence of weight decrease as a reported AE in participants aged 6 to 9 years may be related in part to the higher incidence of anorexia in this age group. The incidence of insomnia was $35.4 \%$ in the younger group and $22.2 \%$ in the older group. When assessed by sex, overall incidence of TEAEs was similar between boys and girls. For some commonly reported TEAEs with LDX treatment (eg, insomnia, headache, and affect lability), incidence was somewhat higher in boys than in girls.

\section{Limitations}

The current analysis has some limitations. As these are post hoc analyses and not part of the a priori planned analyses, they were not adequately powered to make direct comparisons of subgroup differences in efficacy variables or $\mathrm{AE}$ incidence. The relatively small group sizes and the approximately 3:1 ratio of boys to girls in the study make it difficult to form firm conclusions regarding possible sex effects. It should be noted, however, that this sex disparity is improved from previous trials that have shown male-to-female ratios as high as 9:1 [17,44-46]. This improvement may be attributable to better identification and diagnosis of female individuals with ADHD.

While the TEAE tables may appear to show fewer TEAEs during the crossover phase than in the preceding dose-optimization phase, one should consider the relative duration of treatment exposure during the 2 phases, as well as the manner in which TEAEs were reported. TEAE incidence rates are based on newly occurring events at each weekly assessment during the dose-optimization and crossover phases. As such, continuing
TEAEs would only appear at the assessment in which they were first reported. Similarly, when considering the relative incidence of TEAEs during the dose-optimization phase, readers should remember that TEAEs reported by dose may be related to timing of dose escalation during the optimization process rather than to dose-dependent effects. Thus, those TEAEs occurring during the first week of dose-optimization treatment would be reported for $30 \mathrm{mg} / \mathrm{d}$ LDX since all participants began dosing at $30 \mathrm{mg} / \mathrm{d}$ LDX; those TEAEs first occurring during the second week would be ascribed to either $30 \mathrm{mg} / \mathrm{d}$ or $50 \mathrm{mg} / \mathrm{d}$ LDX, and so on. Based on the available data from this study, dose dependence of TEAEs cannot be assessed.

\section{Conclusions}

Although the study was not adequately powered to make direct comparisons of subgroups, the results of this analysis support the efficacy of LDX from 1.5 hours to 13 hours postdose in both boys and girls with medium to large mean effect sizes across the day.

\section{Abbreviations \\ ADHD: attention-deficit/hyperactivity disorder; ADHD-RS-IV: ADHD Rating Scale IV; AE: adverse event; COMACS: Comparison of Methylphenidates in an Analog Classroom Setting; DBP: diastolic blood pressure; DF: degrees of freedom; DSM-IV-TR: Diagnostic and Statistical Manual of Mental Disorders, Fourth Edition, Text Revision; ECG: electrocardiogram; ITT: intention-to-treat; LDX: lisdexamfetamine dimesylate; LS: least squares; MedDRA: Medical Dictionary for Regulatory Activities; MTA: Multimodal Treatment Study of Children With ADHD; MPH-ER: methylphenidate extended-release; OROS- MPH: osmotic-release oral system methylphenidate; PATS: Preschool ADHD Treatment Study; PERMP: Permanent Product Measure of Performance; PERMP-A: PERMP number attempted; PERMP-C: PERMP number correct; SBP: systolic blood pressure; SD: standard deviation; SE: standard error; SKAMP: Swanson, Kotkin, Agler, M-Flynn, and Pelham; SKAMP-A: SKAMP-Attention; SKAMP-D: SKAMP-Deportment; SNAP: Swanson, Nolan and Pelham scale; SWMD: standardized weighted mean difference; TEAE: treatment-emergent adverse event.}

\section{Acknowledgments}

Clinical research was funded by the sponsor, Shire Development Inc. Under the directions of the authors, Asha Philip, PharmD, and Michael Pucci, PhD, employees of Ogilvy CommonHealth Scientific Communications (OCHSC) provided writing assistance for this publication. Editorial assistance in the form of proofreading, copy editing, and fact checking was also provided by OCHSC. Brian Scheckner, PharmD, Brian Dirks, MD, Thomas Babcock, DO, and Michael Nessly also reviewed and edited the manuscript for scientific accuracy. Shire Development Inc. provided funding to OCHSC for support in writing and editing this manuscript. Although the sponsor was involved in the design, collection, analysis, interpretation, and fact checking of information, the content of this manuscript, the ultimate interpretation, and the decision to submit it for publication in Child and Adolescent Psychiatry and Mental Health were made by the authors independently. Authors had direct access to all data and analyses and take full responsibility for this report.

\section{Author details}

${ }^{1}$ University of California, Irvine, Child Development Center, Irvine, California, USA. ${ }^{2}$ Duke University Medical Center, Durham, North Carolina, USA. ${ }^{3}$ Center for Psychiatry and Behavioral Medicine, Las Vegas, Nevada, USA. ${ }^{4}$ Shire Development Inc., Wayne, Pennsylvania, USA. 


\section{Authors' contributions}

SW was an investigator on the parent study and participated in data acquisition, analysis, interpretation, and presentation. SW was fully involved in drafting the manuscript and revising the intellectual content of this manuscript. She has given final approval of this version; SHK was an investigator on the parent study and participated in data acquisition, analysis, interpretation, and presentation. SHK was fully involved in drafting the manuscript and revising the intellectual content of this manuscript. He has given final approval of this version; ACC was an investigator on the parent study and participated in data acquisition, analysis, interpretation, and presentation. ACC was fully involved in drafting the manuscript and revising the intellectual content of this manuscript. She has given final approval of this version. Statistician BA was involved in all post hoc data analysis, interpretation, and presentation. He was fully involved in drafting and revising the intellectual content of this manuscript. BA has given final approval to this version.

\section{Competing interests}

SW, PhD, is a consultant for Abbott, Eli Lilly and Company, McNeil Consumer \& Specialty Pharmaceuticals, Next Wave Pharmaceutical, NIMH, NuTec, Shire US Inc., and Taisho; has received research support from Addrenex, Eli Lilly and Company, McNeil, New River, NICHD, NIMH, Novartis, Otsuka, Psychogenics, Quintiles, Shionogi, and Shire US Inc.; and is a speaker for McNeil Consumer \& Specialty Pharmaceuticals and Shire US Inc; SHK $\mathrm{PhD}$, has received research support and/or consultant fees from Addrenex Pharmaceuticals Inc, CoMentis Inc, the Environmental Protection Agency, the National Institute on Drug Abuse, the National Institute of Environmental Health Sciences, the National Institute of Mental Health, the National Institute of Neurological Disorders and Strokes, Otsuka Pharmaceuticals, Shire Laboratories Inc., and Supernus Pharmaceuticals; ACC, MD, is a consultant for Novartis and Shire; is a speaker for Bristol-Myers Squibb, GlaxoSmithKline, Novartis, and Shire; has received research support from Abbott, Bristol-Myers Squibb, Johnson \& Johnson Pharmaceutical Research \& Development, LLC, Lilly USA, LLC, NextWave, Novartis, Ortho-McNeil-Janssen Scientific Affairs, Shire, and Somerset; BA, MS, is an employee of Shire and holds stock and/or stock options from Shire.

Received: 4 May 2010 Accepted: 14 December 2010 Published: 14 December 2010

\section{References}

1. Pliszka SR, Crismon ML, Hughes CW, Conners CK, Emslie GJ, Jensen PS, McCracken JT, Swanson JM, Lopez M, The Texas Consensus Conference Panel on Pharmacotherapy of Childhood Attention-Deficit/Hyperactivity Disorder: The Texas Children's Medication Algorithm Project: revision of the algorithm for pharmacotherapy of attention-deficit/hyperactivity disorder. J Am Acad Child Adolesc Psychiatry 2006, 45:642-657.

2. Stein MA, Baren M: Welcome progress in the diagnosis and treatment of ADHD in adolescence. Contemp Pediatr 2003, 20:83-110.

3. Faraone SV: Lisdexamfetamine dimesylate: the first long-acting prodrug stimulant treatment for attention deficit/hyperactivity disorder. Expert Opin Pharmacother 2008, 9:1565-1574.

4. Weisler RH: Review of long-acting stimulants in the treatment of attention deficit hyperactivity disorder. Expert Opin Pharmacother 2007, 8:745-758.

5. Stein MA: Innovations in attention-deficit/hyperactivity disorder pharmacotherapy: long-acting stimulant and nonstimulant treatments. Am J Manag Care 2004, 10(suppl):S89-S98.

6. Swanson JM, Wigal SB, Wigal T, Sonuga-Barke E, Greenhill LL, Biederman J, Kollins S, Nguyen AS, DeCory HH, Hirshe Dirksen SJ, Hatch SJ, COMACS Study Group: A comparison of once-daily extended-release methylphenidate formulations in children with attention-deficit/ hyperactivity disorder in the laboratory school (The Comacs Study). Pediatrics 2004, 113:e206-e216.

7. Pennick M: Absorption of lisdexamfetamine dimesylate and its enzymatic conversion to d-amphetamine. Neuropsychiatr Dis Treat 2010, 6:317-327.

8. Biederman J, Krishnan S, Zhang Y, McGough JJ, Findling RL: Efficacy and tolerability of lisdexamfetamine dimesylate (NRP-104) in children with attention-deficit/hyperactivity disorder: a phase III, multicenter, randomized, double-blind, forced-dose, parallel-group study. Clin Ther 2007, 29:450-463.
9. Biederman J, Boellner SW, Childress A, Lopez FA, Krishnan S, Zhang Y: Lisdexamfetamine dimesylate and mixed amphetamine salts extendedrelease in children with ADHD: a double-blind, placebo-controlled, crossover analog classroom study. Biol Psychiatry 2007, 62:970-976.

10. Wigal SB, Kollins SH, Childress AC, Squires L, for the 311 Study Group: A 13hour laboratory school study of lisdexamfetamine dimesylate in schoolaged children with attention-deficit/hyperactivity disorder. Child Adolesc Psychiatry Ment Health 2009, 3:17.

11. Gray JR, Kagan J: The challenge of predicting which children with ADHD respond positively to methylphenidate. J Applied Dev Psychol 2000, 21:471-489.

12. The MTA Cooperative Group: Moderators and mediators of treatment response for children with attention-deficit/hyperactivity disorder: the Multimodal Treatment Study of children with Attention-deficit/ hyperactivity disorder. Arch Gen Psychiatry 1999, 56:1088-1096.

13. Owens EB, Hinshaw SP, Kraemer HC, Arnold LE, Abikoff HB, Cantwell DP, Conners CK, Elliott G, Greenhill LL, Hechtman L, Hoza B, Jensen PS, March JS, Newcorn JH, Pelham WE, Severe JB, Swanson JM, Vitiello B, Wells KC, Wigal T: Which treatment for whom for ADHD? Moderators of treatment response in the MTA. J Consult Clin Psychol 2003, 71:540-552.

14. Sonuga-Barke EJS, Coghill D, Markowitz JS, Swanson JM, Vandenberghe M, Hatch SJ: Sex differences in the response of children with ADHD to once-daily formulations of methylphenidate. J Am Acad Child Adolesc Psychiatry 2007, 46:701-710.

15. Rucklidge JJ: Gender differences in ADHD: implications for psychosocial treatments. Expert Rev Neurother 2008, 8:643-655.

16. Gershon J: A meta-analytic review of gender differences in ADHD. $J$ Atten Disord 2002, 5:143-154.

17. Abikoff HB, Jensen PS, Arnold LL, Hoza B, Hechtman L, Pollack S, Martin D, Alvir J, March JS, Hinshaw S, Vitiello B, Newcorn J, Greiner A, Cantwell DP, Conners CK, Elliott G, Greenhill LL, Kraemer H, Pelham WE Jr, Severe JB, Swanson JM, Wells K, Wigal T: Observed classroom behavior of children with ADHD: relationship to gender and comorbidity. J Abnorm Child Psychol 2002, 30:349-359.

18. Quinn P, Wigal S: Perceptions of girls and ADHD: results from a national survey. MedGenMed 2004, 6:2.

19. Greenhill L, Kollins S, Abikoff H, McCracken J, Riddle M, Swanson J, McGough J, Wigal S, Wigal T, Vitiello B, Skrobala A, Posner K, Ghuman J, Cunningham C, Davies M, Chuang S, Cooper T: Efficacy and safety of immediate-release methylphenidate treatment for preschoolers with ADHD. J Am Acad Child Adolesc Psychiatry 2006, 45:1284-1293.

20. Greenhill LL, Swanson JM, Vitiello B, Davies M, Clevenger W, Wu M, Arnold LE, Abikoff HB, Bukstein OG, Conners CK, Elliott GR, Hechtman L, Hinshaw SP, Hoza B, Jensen PS, Kraemer HC, March JS, Newcorn JH, Severe JB, Wells K, Wigal T: Impairment and deportment responses to different methylphenidate doses in children with ADHD: the MTA titration trial. J Am Acad Child Adolesc Psychiatry 2001, 40:180-187.

21. Wigal T, Greenhill L, Chuang S, McGough J, Vitiello B, Skrobala A, Swanson J, Wigal S, Abikoff H, Kollins S, McCracken J, Riddle M, Posner K, Ghuman J, Davies M, Thorp B, Stehli A: Safety and tolerability of methylphenidate in preschool children with ADHD. J Am Acad Child Adolesc Psychiatry 2006, 45:1294-1303.

22. Wigal SB, Gupta S, Greenhill L, Posner K, Lerner M, Steinhoff K, Wigal T, Kapelinski A, Martinez J, Modi NB, Stehli A, Swanson J: Pharmacokinetics of methylphenidate in preschoolers with attention-deficit/hyperactivity disorder. J Child Adolesc Psychopharmacol 2007, 17:153-164.

23. Stein MA, Sarampote CS, Waldman ID, Robb AS, Conlon C, Pearl PL, Black DO, Seymour KE, Newcorn JH: A dose-response study of OROS methylphenidate in children with attention-deficit/hyperactivity disorder. Pediatrics 2003, 112:e404.

24. Faraone SV: Using meta-analysis to compare the efficacy of medications for attention-deficit/hyperactivity disorder in youths. P\&T 2009, 34:678-694.

25. Wigal SB, Gupta S, Guinta D, Swanson JM: Reliability and validity of the SKAMP rating scale in a laboratory school setting. Psychopharmacol Bull 1998, 34:47-53.

26. Wigal SB, Wigal TL: The laboratory school protocol: its origin, use, and new applications. J Atten Disord 2006, 10:92-111.

27. DuPaul GJ, Power TJ, Anastopoulos AD, Reid R: ADHD Rating Scale-IV: Checklists, Norms, and Clinical Interpretation. New York, NY: Guilford Press; 1998. 
28. Curtin F, Altman DG, Elbourne D: Meta-analysis combining parallel and cross-over clinical trials. I: Continuous outcomes. Stat Med 2002, 21:2131-2144.

29. McGough JJ, Faraone SV: Estimating the size of treatment effects: moving beyond $\mathrm{p}$ values. Psychiatry (Edgmont) 2009, 6:21-29.

30. Cohen J: Quantitative methods in psychology: a power primer. Psychol Bull 1992, 112:155-159.

31. Becker JB: Gender differences in dopaminergic function in striatum and nucleus accumbens. Pharmacol Biochem Behav 1999, 64:803-812.

32. Munro CA, McCaul ME, Wong DF, Oswald LM, Zhou Y, Brasic J, Kuwabara H, Kumar A, Alexander M, Ye W, Wand GS: Sex differences in striatal dopamine release in healthy adults. Biol Psychiatry 2006, 59:966-974.

33. Biederman J, Mick E, Faraone SV: Age-dependent decline of symptoms of attention deficit hyperactivity disorder: impact of remission definition and symptom type. Am J Psychiatry 2000, 157:816-818

34. Hart EL, Lahey BB, Loeber R, Applegate B, Frick PJ: Developmental change in attention-deficit hyperactivity disorder in boys: a four-year longitudinal study. J Abnorm Child Psychol 1995, 23:729-749.

35. Todd RD, Huang H, Todorov AA, Neuman RJ, Reiersen AM, Henderson CA, Reich WC: Predictors of stability of attention-deficit/hyperactivity disorder subtypes from childhood to young adulthood. J Am Acad Child Adolesc Psychiatry 2008, 47:76-85.

36. Faraone $\mathrm{S}$, Schreckengost J: Lisdexamfetamine dimesylate effect size in children with attention-deficit/hyperactivity disorder. Poster presented at: American Academy of Child \& Adolescent Psychiatry's 54th Annual Meeting; October 23-28, 2007, Boston, MA .

37. Biederman J, Faraone SV: Attention-deficit hyperactivity disorder. Lancet 2005, 366:237-248

38. Smith BH, Pelham WE, Gnagy E, Yudell RS: Equivalent effects of stimulant treatment for attention-deficit hyperactivity disorder during childhood and adolescence. J Am Acad Child Adolesc Psychiatry 1998, 37:314-321.

39. McCracken JT, Biederman J, Greenhill LL, Swanson JM, McGough JJ, Spencer TJ, Posner K, Wigal S, Pataki C, Zhang Y, Tulloch S: Analog classroom assessment of a once-daily mixed amphetamine formulation SLI381 (Adderall XR), in children with ADHD. J Am Acad Child Adolesc Psychiatry 2003, 42:673-683.

40. Pierce D, Dixon CM, Wigal SB, McGough JJ: Pharmacokinetics of methylphenidate transdermal system (MTS): results from a laboratory classroom study. J Child Adolesc Psychopharmacol 2008, 18:355-364.

41. Krishnan SM, Stark JG: Multiple daily-dose pharmacokinetics of lisdexamfetamine dimesylate in healthy adult volunteers. Curr Med Res Opin 2008, 24:33-40.

42. Biederman J, Lopez FA, Boellner SW, Chandler MC: A randomized, doubleblind, placebo-controlled, parallel-group study of SLI381 (Adderall XR) in children with attention-deficit/hyperactivity disorder. Pediatrics 2002, 110:258-266.

43. Wigal S, Swanson JM, Feifel D, Sangal RB, Elia J, Casat CD, Zeldis JB, Conners CK: A double-blind, placebo-controlled trial of dexmethylphenidate hydrochloride and $d, l$-threo-methylphenidate hydrochloride in children with attention-deficit/hyperactivity disorder. $J$ Am Acad Child Adolesc Psychiatry 2004, 43:1406-1414.

44. Arnold LE: Sex differences in ADHD: conference summary. J Abnorm Child Psychol 1996, 24:555-569

45. Barbaresi WJ, Katusic SK, Colligan RC, Weaver AL, Jacobsen SJ: Long-term school outcomes for children with attention-deficit/hyperactivity disorder: a population-based perspective. J Dev Behav Pediatr 2007 , 28:265-273.

46. Werry JS, Aman MG, Diamond E: Imipramine and methylphenidate in hyperactive children. J Child Psychol Psychiatry 1980, 21:27-35.

doi:10.1186/1753-2000-4-32

Cite this article as: Wigal et al:: Efficacy and tolerability of lisdexamfetamine dimesylate in children with attention-deficit/ hyperactivity disorder: sex and age effects and effect size across the day. Child and Adolescent Psychiatry and Mental Health 2010 4:32.

\section{Submit your next manuscript to BioMed Central and take full advantage of:}

- Convenient online submission

- Thorough peer review

- No space constraints or color figure charges

- Immediate publication on acceptance

- Inclusion in PubMed, CAS, Scopus and Google Scholar

- Research which is freely available for redistribution

Submit your manuscript at www.biomedcentral.com/submit
Ciomed Central 